\title{
Module bundles and module amenability
}

\author{
Terje Hill and David A. Robbins
}

\begin{abstract}
Let $X$ be a compact Hausdorff space, and let $\left\{A_{x}: x \in X\right\}$ and $\left\{B_{x}: x \in X\right\}$ be collections of Banach algebras such that each $A_{x}$ is a $B_{x}$-bimodule. Using the theory of bundles of Banach spaces as a tool, we investigate the module amenability of certain algebras of $A_{x}$-valued functions on $X$ over algebras of $B_{x}$-valued functions on $X$.
\end{abstract}

\section{Introduction}

Let $X$ be a set, perhaps even a topological space, and suppose that $\left\{E_{x}: x \in X\right\}$ is a collection of Banach spaces indexed by $X$. Let $\mathcal{S}$ be a Banach space of choice functions $\sigma$ from $X$ to the disjoint union $\mathcal{E}=\dot{\bigcup}\left\{E_{x}: x \in X\right\}$ of the $E_{x}$ (i.e., $\sigma \in \prod_{x \in X} E_{x}$ ) such that $\{\sigma(x): \sigma \in \mathcal{S}\}=E_{x}$ for each $x \in X$. If $\mathcal{S}$ has a property $\mathcal{P}$ whenever each $E_{x}$ has property $\mathcal{P}$ (or perhaps when only some do), we say that $\mathcal{P}$ is hereditary for $\mathcal{S}$. It seems fairly natural to ask for which spaces $\mathcal{S}$ and properties $\mathcal{P}$ this hereditary condition holds.

In this paper, when the $E_{x}$ are Banach algebras, we will investigate the extent to which a condition sufficient for module amenability is hereditary for spaces $\mathcal{S}$ of a specific description. We will conduct this investigation using the language of bundles of Banach spaces ( = Banach bundles) and their Banach section spaces. The paper is organized as follows. In Section 2 we will introduce the notion of Banach bundles and from the somewhat scattered references we will gather together for the reader's convenience some definitions, useful facts, and examples. We close the section with a very abbreviated bibliography of Banach bundles. In Section 3 we will discuss the notion of module amenability, and prove our main result. In the very

Received January 28, 2021.

2020 Mathematics Subject Classification. Primary 46H25; Secondary 46H10.

Key words and phrases. Bundles of Banach algebras, bundles of Banach modules, module amenability.

https://doi.org/10.12697/ACUTM.2021.25.08 
short Section 4 we will prove a result regarding irreducibility of modules over section spaces of bundles of Banach algebras, and make two auxiliary observations.

\section{Banach bundles - introduction, examples, and relevant properties}

We first describe the spaces $\mathcal{S}$ with which we will be working. Let $X$ be a compact Hausdorff space and, as above, let $\left\{E_{x}: x \in X\right\}$ be a collection of Banach spaces indexed by $X$, with disjoint union $\mathcal{E}=\bigcup \dot{\bigcup}\left\{E_{x}: x \in X\right\}$. Let $\mathbb{K}$ be the common scalar field, either $\mathbb{R}$ or $\mathbb{C}$, and let $\pi: \mathcal{E} \rightarrow X$ be the natural projection. We let $\mathcal{S}$ be a vector space of choice functions $\sigma: X \rightarrow \mathcal{E}$ which satisfies the following:

1) for each $x \in X, E_{x}=\phi_{x}(\mathcal{S})=\{\sigma(x): \sigma \in \mathcal{S}\}$ ( $\mathcal{S}$ is said to be full; $\phi_{x}$ is the evaluation map at $x$ );

2) for each $\sigma \in \mathcal{S}$ the norm map $x \mapsto\|\sigma(x)\|$ is upper semicontinuous;

3) $\mathcal{S}$ is a $C(X)$-module under the pointwise operations;

4) $\mathcal{S}$ is closed in the sup-norm.

(We observe that, while we are operating in the context of Banach spaces and algebras only, it is possible using only 1) and 3) above to describe an analogous situation in the more general context where the $E_{x}$ are arbitrary topological algebras; see [1].)

Note that in the presence of the sup-norm on the space $\mathcal{S}$, it is in fact a $C(X)$-locally convex module. That is (among other equivalent definitions, see [22] or [17]), whenever $f, g \in C(X)$ with $f g=0$, we have $\|(f+g) \sigma\|=\max \{\|f \sigma\|,\|g \sigma\|\}$ for all $\sigma \in \mathcal{S}$.

When $\mathcal{S}$ satisfies these conditions, and in the presence of the compactness of $X$ (in the language of [11], $\mathcal{S}$ is a function module), there is a topology on $\mathcal{E}$ such that $\mathcal{S}$ is precisely the space of continuous choice functions $\sigma: X \rightarrow \mathcal{E}$. This space of continuous choice functions is denoted by $\Gamma(\pi)$, which is the section space of the bundle of Banach spaces (= Banach bundle) $\pi: \mathcal{E} \rightarrow X$ with fibers $E_{x}$; we shall denote this bundle by $\pi$ when there can be no confusion. The topology on $\mathcal{E}$ is called the bundle topology, and is defined by tubes of the form

$$
\mathcal{T}=\mathcal{T}(U, z, \varepsilon)=\{w \in \mathcal{E}: \pi(w) \in U \text { and }\|\sigma(\pi(w))-w\|<\varepsilon\},
$$

where $\sigma \in \mathcal{S}$ is such that $\sigma(x)=z \in E_{x}, U \subset X$ varies over neighborhoods of $x \in X$, and $\varepsilon>0$ varies; the tubes $\mathcal{T}$ form a subbasis for the bundle topology. With this bundle topology, the addition map $(z, w) \mapsto z+w$ is continuous from $\mathcal{E} \vee \mathcal{E}$ to $\mathcal{E}$, where $\mathcal{E} \vee \mathcal{E}=\bigcup \dot{\bigcup}\left\{E_{x} \times E_{x}: x \in X\right\}$ is the fibered product of $\mathcal{E}$ with itself with the relative topology from $\mathcal{E} \times \mathcal{E}$. The intuitive notion is that if $\sigma \in \Gamma(\pi)$, then we can think of $\sigma(x)$ as moving continuously through the various spaces $E_{x}$ as $x$ moves continuously through $X$. 
Two sources which the reader may find most immediately useful for information on the development of the theory of Banach bundles are [17] and [30].

The definition above of a Banach bundle then leads to the reasonably natural definition of a bundle of Banach algebras. Suppose that $\pi: \mathcal{A}=\bigcup_{x \in X}\left\{A_{x}: x \in X\right\} \rightarrow X$ is a Banach bundle where each $A_{x}$ is a Banach algebra. As noted above, with the bundle topology on $\mathcal{A}$, the map $(z, w) \mapsto z+w, \mathcal{A} \vee \mathcal{A} \rightarrow \mathcal{A}$ is continuous. If in addition $\Gamma(\pi)$ is closed under pointwise multiplication, then we call $\pi$ a bundle of Banach algebras; this is equivalent to the continuity of the multiplication map $(z, w) \mapsto z w$ from $\mathcal{A} \vee \mathcal{A}$ to $\mathcal{A}$. If each $A_{x}$ has identity $e_{x}$, and if the identity selection $e$ (given by $e(x)=e_{x}$ ) is actually in $\Gamma(\pi)$, we say that $\pi$ is an algebra bundle with identity. (Even when each fiber $A_{x}$ of $\mathcal{A}$ has an identity, $\mathcal{A}$ may yet not be a bundle with identity. For example, we may identify $c_{0}([0,1])$ with the section space $\Gamma\left(\pi_{0}\right)$ of the "spiky" bundle $\pi_{0}: \mathcal{A}=\dot{\bigcup}\{\mathbb{K}: x \in[0,1]\} \rightarrow[0,1]$ with constant fiber $\mathbb{K}$. Evidently, each fiber $\mathbb{K}_{x}=\mathbb{K}$ has an identity, but the identity selection is not in $\Gamma(\pi)$. See [30].)

Given the notion of a bundle of Banach algebras, then it should be clear what we mean by a bundle of Banach modules over a bundle of Banach algebras. Let $\pi$ be a bundle of Banach algebras $A_{x}$ with section space $\Gamma(\pi)$, as above. Suppose that for each $x \in X, M_{x}$ is a left (right, bi-) module over $A_{x}$, and that $\rho: \mathcal{M}=\dot{\bigcup}\left\{M_{x}: x \in X\right\} \rightarrow X$ is a bundle of Banach spaces such that for each $\tau \in \Gamma(\rho)$ and $\alpha \in \Gamma(\pi)$ we have $\alpha \tau \in \Gamma(\rho)$. (This is equivalent to the continuity of the map $(z, w) \mapsto z w$ from $\mathcal{A} \vee \mathcal{M}$ to $\mathcal{M}$.) Then $\rho$ is a bundle of left Banach modules over the bundle $\pi$ of Banach algebras. Evidently, the same notions apply for right and bimodules. If the algebra bundle $\pi$ has identity, and if each $M_{x}$ is unital as an $A_{x}$-module, then clearly $\Gamma(\rho)$ is unital as a $\Gamma(\pi)$-module. See [31] for this development.

Our investigation in Section 3 into how a condition sufficient for module amenability might be hereditary is motivated by similar cases of Banach bundles (of algebras, or just of spaces) $\pi: \mathcal{E} \rightarrow X$, where properties of the $E_{x}$ can be inherited by $\Gamma(\pi)$. For example, in [16] it was shown that $\Gamma(\pi)$ has the approximation property if and only if each fiber $E_{x}$ does also. More recently, the present authors showed in [25] that if $\pi$ is a Banach algebra bundle with each fiber commutative, then $\Gamma(\pi)$ satisfies spectral synthesis if and only if each fiber does also. The papers [38] and [23] show that several geometric properties of the $E_{x}$ can be inherited by $\Gamma(\pi)$. The authors also showed, in [26], that if $\pi$ is a bundle of Banach algebras, then $\Gamma(\pi)$ is amenable if and only if the fibers $E_{x}$ are "uniformly amenable" in a very reasonable definition of the term (see the reference for the meaning of this). The present paper continues in this vein. (We also note here the 
importance for the notion of heredity of using the sup-norm on function spaces $\mathcal{S}$ as described at the beginning of this section. For example, it is evident that $\mathcal{S}=\ell^{1}([0,1])$, when regarded as a space of functions from $[0,1]$ to $\bigcup\left\{\mathbb{K}_{x}=\mathbb{K}: x \in[0,1]\right\}$, satisfies conditions 1$)$ - 3) above; clearly each $\mathbb{K}_{x}$ has a bounded approximate identity, but $\ell^{1}([0,1])$ does not.)

Following are some examples of module bundles over algebra bundles. Here and elsewhere in the paper we will abuse notation slightly in the following fashion. Suppose that $A$ is a Banach algebra, that $I \subset A$ is a closed ideal, and that $M$ is a (left) $A$-module. Unless otherwise noted, by $I M$ (or by $I \cdot M$, if desirable for typographic clarity) we will mean the closure in $M$ of the set $\{a m: a \in I, m \in M\}$. If $M=A M$, we say that $M$ is an essential $A$-module.

Example 1. Any Banach space over $\mathbb{K}$ is also a module over $\mathbb{K}$. Let $\rho: \mathcal{E}=\dot{\bigcup}\left\{E_{x}: x \in X\right\} \rightarrow X$ be a Banach bundle, with each $E_{x}$ a Banach space over a common $\mathbb{K}$, and let $\pi: \mathcal{A}=X \times \mathbb{K} \rightarrow X$ be the trivial bundle, where $\mathcal{A}$ has its product topology and $\Gamma(\pi) \simeq C(X)$. Then $\Gamma(\rho)$ satisfies conditions 1$)-4)$, so that $\rho$ is a bundle of modules over $\pi$.

Example 2. Let $E$ be a Banach module over the Banach algebra $A$, and let $C(X, E)$ be the space of continuous $E$-valued functions. Then $C(X, E)$ is a Banach module over $C(X, A)$. We have $C(X, E) \simeq \Gamma(\rho)$, where $\rho: X \times E \rightarrow X$ is the trivial bundle with constant fiber $E$ and $X \times E$ has the product topology; $\rho$ is a bundle of Banach modules over the trivial bundle $\pi: X \times A \rightarrow X$ (defined similarly).

Example 3. Suppose that for each $x \in X, E_{x}$ is a Banach module over the algebra $A_{x}$. Denote by $\rho_{0}: \mathcal{E} \rightarrow X$ the spiky bundle with fibers $E_{x}$ over $X$ (again, see [30]). Then $\Gamma\left(\rho_{0}\right)$ is the closure in the sup norm of the space of choice functions with finite support. If $\pi: \mathcal{A} \rightarrow X$ is any algebra bundle with fibers $A_{x}$, then $\rho_{0}$ is a module bundle over $\pi$.

Example 4. Let $A$ be a Banach algebra, and let $\mathcal{I}$ be a collection of closed ideals $I \subset A$. Suppose that $\mathcal{I}$ can be topologized as a compact Hausdorff space in such a way that the map $I \mapsto\|a+I\|$ from $\mathcal{I}$ to $\mathbb{R}^{+}$is upper semicontinuous for each $a \in A$. (In the language of [30], $\mathcal{I}$ is said to be topologically compatible with $A$.) Let $M$ be a Banach $A$-module. For $I \in \mathcal{I}$, set $F_{I}=M / I M$, and let $\mathcal{F}=\bigcup \grave{\bigcup}\left\{F_{I}: I \in \mathcal{I}\right\}$. Then $I \mapsto\|m+I M\|$ is upper semicontinuous for each $m \in M$, so that $\rho: \mathcal{F} \rightarrow X$ is a Banach module bundle over the Banach algebra bundle $\pi: \mathcal{A}=\dot{\bigcup}\{A / I: I \in \mathcal{I}\} \rightarrow X$. In particular, if $A$ is commutative with identity, and $\mathcal{I}$ is the collection of maximal ideals ( $=$ kernels of $\mathbb{K}$-valued algebra homomorphisms on $A$ ) in $A$, with its weak*-topology, and if $M$ is an $A$-module, then $I \mapsto\|a+I\|$ 
and $I \mapsto\|m+I M\|$ are upper semicontinuous for each $a \in A$ and $m \in M$, and so $\rho$ and $\pi$ satisfy the described conditions. (See [31].) Or, for another example in this vein, the Dauns-Hofmann representation theorem (see, e.g., [21]) states that a $C^{*}$-algebra is isometrically isomorphic to the section space of a certain bundle of (quotient) $C^{*}$-algebras.

Following are some salient facts regarding Banach bundles (algebra bundles, module bundles) which we will use in Section 3 in proving the hereditary nature of module amenability (to be defined therein).

I) If $\pi: \mathcal{E} \rightarrow X$ is a Banach bundle, and $F \subset \Gamma(\pi)$ is a closed linear subspace which is also a $C(X)$-module, set $F_{x}=\{\sigma(x): \sigma \in F\}=\phi_{x}(F)$, where $\phi_{x}$ is the evaluation map at $x$; clearly $F_{x} \subset E_{x}$. If we set $\left(F_{x}\right)^{x}=\left\{\sigma \in \Gamma(\pi): \sigma(x) \in F_{x}\right\}$, then $F=\bigcap_{x \in X} \overline{\left(F_{x}\right)^{x}}$. The subspace and $C(X)$-module $F$ is itself the section space of a bundle $\rho_{1}: \mathcal{F} \rightarrow X$ with fibers $F_{x}$. If $\sigma \in \Gamma(\pi)$, then $\sigma \in F$ if and only if $\sigma(x) \in F_{x}$ for all $x$. Moreover, there is a bundle $\pi_{1}: \mathcal{E} / \mathcal{F} \rightarrow X$ whose fibers over $x$ are (isometrically isomorphic to) $E_{x} / F_{x}$, and an isometric $C(X)$-isomorphism $\Phi: \Gamma(\pi) / \Gamma\left(\rho_{1}\right)=\Gamma(\pi) / F \rightarrow \Gamma\left(\pi_{1}\right)$, with the correspondence given by $[\Phi(\sigma+F)](x)=\sigma(x)+F_{x}$; we have $\|\sigma+F\|=\sup _{x \in X}\left\|\sigma(x)+F_{x}\right\|$.

II) If $\pi: \mathcal{E} \rightarrow X$ is a Banach bundle, and $U \subset X$ is a closed subset, let $I_{U} \subset C(X)$ denote the ideal of functions which vanish on $U$. Then, as a special case of $\mathrm{I})$, we have $\Gamma\left(\pi_{U}\right) \simeq \Gamma(\pi) / I_{U} \Gamma(\pi)$, where $\Gamma\left(\pi_{U}\right)$ is the section

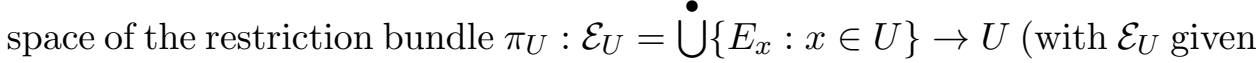
its relative topology from $\mathcal{E}$ ). Because of the Tietze theorem for bundles (see, e.g., [29, Lemma 3.3] or [17, Corollary 4.5]), we can identify the restriction bundle $\pi_{U}$ with its extension bundle $\pi_{U}^{\prime}: \mathcal{E}^{\prime} \rightarrow X$, where $E_{x}^{\prime}=0$ if $x \notin U$ and $E_{x}^{\prime}=E_{x}$ if $x \in U$. Even more particularly, if $I_{x} \subset C(X)$ is the maximal ideal of functions in $C(X)$ which vanish at $x$, we have $E_{x} \simeq \Gamma(\pi) / I_{x} \Gamma(\pi)$ as a Banach space (and as the highly restricted "single point" section space $\Gamma\left(\pi_{x}^{\prime}\right)$ of the bundle $\pi_{x}^{\prime}: \mathcal{E}_{x}^{\prime} \rightarrow X$, whose fibers are $E_{y}^{\prime}=0$ if $y \neq x$, and $\left.E_{x}^{\prime}=E_{x}\right)$.

III) Let $\pi: \mathcal{E} \rightarrow X$ and $\rho: \mathcal{F} \rightarrow X$ be Banach bundles, and let $T: \Gamma(\pi) \rightarrow \Gamma(\rho)$ be a continuous $C(X)$-homomorphism, i.e., $T(f \sigma)=f T(\sigma)$ for $f \in C(X)$ and $\sigma \in \Gamma(\pi)$. Then there exist induced linear maps $T_{x}: E_{x} \rightarrow F_{x}$ which make this diagram commute:

$$
\begin{array}{ccc}
\Gamma(\pi) & \rightarrow & \Gamma(\rho) \\
\downarrow & & \downarrow \\
E_{x} & \longrightarrow & F_{x}
\end{array},
$$

where the downward arrows indicate evaluation of sections at $x \in X$. It can be shown that $\|T\|=\sup _{x}\left\|T_{x}\right\|$, and that $T$ induces a continuous map 
$\widetilde{T}: \mathcal{E} \rightarrow \mathcal{F}$ which makes this diagram commute:

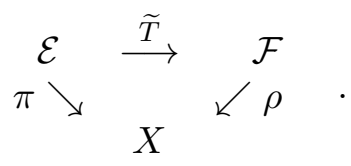

(Figure 2)

Conversely, each continuous map $\widetilde{T}: \mathcal{E} \rightarrow \mathcal{F}$ which makes the diagram in Figure 2 commute and such that $(\widetilde{T})_{x}: E_{x} \rightarrow F_{x}$ is linear (where $(\widetilde{T})_{x}$ is the restriction of $(\widetilde{T})_{x}$ to $\left.E_{x}\right)$ describes a $C(X)$-homomorphism $T: \Gamma(\pi) \rightarrow \Gamma(\rho)$, defined by $(T \sigma)(x)=(\widetilde{T})_{x}(\sigma(x))$.

IV) Recall from the beginning of this section that, if $M$ is a $C(X)$-module, we say that $M$ is $C(X)$-locally convex provided that (among other characterizations) we have $\|(f+g) m\|=\max \{\|f m\|,\|g m\|\}$ whenever $f, g \in C(X)$ with $f g=0$, and $m \in M$. A $C(X)$-locally convex module $M$ is isometrically $C(X)$-isomorphic to the section space $\Gamma(\pi)$ of a bundle $\pi: \mathcal{E}=\dot{\bigcup} E_{x} \rightarrow X$, where $E_{x}=M / I_{x} M$. Conversely, each such section space is $C(X)$-locally convex.

Of these Remarks, I) and II) can be found in the discussion in Chapter 9 of [17]; III) can be found in [30]; and IV) can be found in [17], Chapter 7.

We close this section with the following bibliographical note, taken in large part from [24]. Banach bundles and their section spaces have been around under a variety of labels for some time, to a variety of ends. The terminology includes "upper semicontinuous function modules" [11], as noted above; "Banach function modules" [7]; and "bundles of topological vector spaces" [17]. A more categorical discussion of the matter can be found in, e.g., [22] and [32]; the paper [19] and some of its references are also relevant. Indeed, the basic ideas go back at least as far as 1949 [18] and 1951 [28], under the terminology "continuous sums" (of Banach spaces). The tie between Banach bundles and function modules can be summarized by the following. When $X$ is compact, the section space of any Banach bundle is a function module, and, conversely, any Banach $C(X)$-module $M$ has a norm-decreasing $C(X)$-module representation as the space of sections of a certain "canonical" bundle $\pi: \mathcal{E} \rightarrow X$ with fibers $E_{x}=M / I_{x} M$, where again $I_{x} M$ is the closed span of $\{f m: f \in C(X)$ and $f(x)=0, m \in M\}$ (see [30]) - and, if $M$ is $C(X)$-locally convex, this representation is an isometric $C(X)$-isomorphism; this is the content of IV) above. See [15], and also [12] and [14] for more connections, especially regarding bundles of algebras.

\section{Module amenability and heredity}

Let $A$ and $B$ be Banach algebras, where $A$ is also a Banach $B$-bimodule. The notion of module amenability (of $A$ as a $B$-bimodule) was introduced by M. Amini in [2] and its Corrigendum [3] as a generalization of the classic 
concept of amenability for Banach algebras (see [27]) with the goal of using module amenability as a tool for studying certain aspects of semigroup algebras. It was subsequently found that the antecedent conditions on $A$ and $B$ which were used in [2] and [3] were inadequate to the purpose. Those conditions were modified in [35] and [10], and have been used in a sizeable number of papers by Amini and others since that time ([4]). (See, e.g., [8], [9], [36], [6] or [5].) The actual definition of module amenability as introduced in [2] has not changed over time. It is already known that, loosely speaking and just as in the classical case of amenable Banach algebras, module amenability is preserved by quotients and tensor products. (See various of the papers cited above.) Our purpose is to investigate how the property of module amenability (actually, a property sufficient for it), with the antecedent conditions on $A$ and $B$ from [35] and [10] et seq., is hereditary for appropriately specified spaces of algebra-valued functions. We now provide specifics.

Definition 1 (see, e.g., [35] and [10]). Suppose that $A$ and $B$ are Banach algebras such that $A$ is a Banach $B$-bimodule, and let $M$ be simultaneously a Banach $A$ - and $B$-bimodule. Suppose that the following compatibility conditions of actions (of $B$ acting on $A$ ) are satisfied:

$$
\left(^{*}\right) b\left(a a^{\prime}\right)=(b a) a^{\prime} \text { and }\left(^{* *}\right)\left(a a^{\prime}\right) b=a\left(a^{\prime} b\right), \quad a, a^{\prime} \in A, b \in B .
$$

Suppose also that

$$
b(a m)=(b a) m, a(b m)=(a b) m \text { and }(b m) a=b(m a)
$$

for all $a \in A, b \in B$, and $m \in M$ for the left actions of $A$ and $B$ on $M$, and similarly for the right and two-sided actions of $A$ and $B$ on $M$. We then call $M$ a Banach $A-B$ module.

If additionally we have

$$
b m=m b
$$

for all $b \in B$ and $m \in M$, we say that $M$ is a commutative $A$ - $B$ module.

Note that the commutativity of $M$ as an $A-B$ bimodule specifically refers to the action of $B$ on $M$, and that $A$ itself may not be an $A-B$ module (although if $A$ is a commutative $B$-module it will be so).

If $M$ is a commutative $A$ - $B$ bimodule, then so is the dual space $M^{*}$, where the multiplication is given as usual by

$$
(f a)(m)=f(a m),(a f)(m)=f(m a)
$$

and

$$
(f b)(m)=f(b m)=f(m b)=(b f)(m)
$$

for $f \in M^{*}, a \in A, b \in B, m \in M$. 
Let $M$ be an $A-B$ bimodule. Then an $A-B$ module derivation of $A$ is a continuous linear map $D: A \rightarrow M$ which satisfies the properties

$$
D\left(a a^{\prime}\right)=D(a) a^{\prime}+a D\left(a^{\prime}\right)
$$

and

$$
D(b a)=b D(a), D(a b)=D(a) b
$$

for $a, a^{\prime} \in A, b \in B$. Note that $D$ is a $B$-module map of $A$ to $M$. If $M$ is a commutative $A$ - $B$ bimodule and $m \in M$, define the map $\delta_{m}: A \rightarrow M$ by

$$
\delta_{m}(a)=a m-m a \text { for } a \in A \text { and } m \in M .
$$

It is straightforward to check that in the presence of the commutativity of $M$ and the compatibility conditions given in conditions (1) and (2) above, $\delta_{m}$ is an $A$ - $B$ module derivation. We call a map of the form $\delta_{m}$ an inner derivation.

We then make the following definition, originally appearing in [2] and [3], and repeated in [35] and subsequent papers.

Definition 2. Let $A$ and $B$ be Banach algebras, and suppose that $A$ is also a $B$-bimodule satisfying (*) of condition (1). Then $A$ is said to be $B$-module amenable ( = amenable as a $B$-module) if, whenever $M$ is a commutative $A-B$ module satisfying condition (2) and $D: A \rightarrow M^{*}$ is an $A-B$ module derivation, then $D$ is inner, i.e., $D=\delta_{m^{*}}$ for some $m^{*} \in M^{*}$.

Although we will not be using this homological characterization, we note that this definition is equivalent to asserting that $\mathcal{H}_{B}^{1}\left(A, M^{*}\right)=0$ for each commutative $A$ - $B$ module $M$, where $\mathcal{H}_{B}^{1}\left(A, M^{*}\right)$ denotes the first cohomology group, relative to $B$, with coefficients in $M^{*}$.

In the case that $B=\mathbb{K}$, any Banach $A$-bimodule $M$ is automatically a commutative $A$ - $\mathbb{K}$ bimodule, and the definition of $B$-module amenability of $A$ reduces to the usual notion of amenability of a Banach algebra (see [27]).

Now, let $A$ be a $B$-bimodule, and also consider $A$ as a bimodule over itself. Then the projective tensor product $A \widehat{\otimes} A$ is both an $A$ - and a $B$-bimodule under the actions $b\left(a \otimes a^{\prime}\right)=(b a) \otimes a^{\prime}$ and $c\left(a \otimes a^{\prime}\right)=(c a) \otimes a^{\prime}$, and similarly for the right actions of $B$ and $A$ on $A \widehat{\otimes} A$, where $a, a^{\prime}, c \in A$ and $b \in B$. We let $K_{B}$, the $B$-balanced kernel in $A \widehat{\otimes} A$ be the closed span in $A \widehat{\otimes} A$ of elements of the form $(a b) \otimes a^{\prime}-a \otimes\left(b a^{\prime}\right)$, and set $A \otimes_{B} A:=(A \widehat{\otimes} A) / K_{B}$, as in [37]. For $a, a^{\prime} \in A$, we will write an elementary element of $A \otimes_{B} A$ as $a \otimes_{B} a^{\prime}=a \otimes a^{\prime}+K_{B}$, and use either as convenient for clarity.

Define the map $\omega: A \widehat{\otimes} A \rightarrow A$ by $\omega\left(a \otimes a^{\prime}\right)=a a^{\prime}$, and let $J_{B}$ be the closure in $A$ of $\omega\left(K_{B}\right)$. Then $\omega$ induces a map

$$
\widetilde{\omega}: A \otimes_{B} A \rightarrow A / J_{B}, a \otimes a^{\prime}+K_{B} \mapsto a a^{\prime}+J_{B},
$$


as in this diagram:

$$
\begin{array}{cccc}
A \widehat{\otimes} A & \stackrel{\omega}{\longrightarrow} & A \\
& \downarrow & & \downarrow \\
A \otimes_{B} A=(A \widehat{\otimes} A) / K_{B} & \longrightarrow & A / J_{B}
\end{array} .
$$

All maps involved are norm-decreasing. We have that $K_{B}$ is an $A$ - and a $B$ submodule of $A \widehat{\otimes} A$, and $J_{B}$ is an $A$ - and a $B$-submodule of $A$; hence $A \otimes_{B} A$ and $A / J_{B}$ are both $A$ - and $B$-modules, and $A / J_{B}$ is an $A$ - $B$ module. (See [35]).

For the following definition, recall that if $B$ is a Banach algebra, and if $M$ is a Banach $B$-bimodule, we say that a net $\left\{b_{i}\right\} \subset B$ is a (two-sided) approximate identity for $M$ if both $b_{i} m \rightarrow m$ and $m b_{i} \rightarrow m$ in norm in $M$; $\left\{b_{i}\right\}$ is a bounded approximate identity if for some $q>0$ we have $\left\|b_{i}\right\|<q$ for all $i$; left- and right-approximate identities are defined analogously.

Definition 3 (see [35]). Let $A$ be a $B$-bimodule satisfying condition (1). The algebra $A$ is said to have a $B$-module approximate diagonal if there is a bounded net $\left\{u_{\lambda}\right\} \subset A \otimes_{B} A$ such that

1) $\left\{\widetilde{\omega}\left(u_{\lambda}\right)\right\}$ is a bounded approximate identity (b.a.i.) for $A / J_{B}$ (that is, for each $a \in A$ we have

$$
\left\|\left[\widetilde{\omega}\left(u_{\lambda}\right) a-a\right]+J_{B}\right\| \rightarrow 0 \text { and }\left\|\left[a \widetilde{\omega}\left(u_{\lambda}\right)-a\right]+J_{B}\right\| \rightarrow 0,
$$

where the norms are taken in $\left.A / J_{B}\right)$; and

2) for each $a \in A$, we have $\left\|u_{\lambda} a-a u_{\lambda}+K_{B}\right\| \rightarrow 0$ (where the norm is taken in $\left.A / K_{B}\right)$.

In 2), the multiplication is given by, for example, if $u_{\lambda}=\sum c_{k} \otimes c_{k}^{\prime}+K_{B}$, then $u_{\lambda} a=\sum c_{k} \otimes\left(c_{k}^{\prime} a\right)+K_{B}$.

The following establishes the fundamental relationship between the $B$-module amenability of $A$ and the existence of a $B$-module approximate diagonal when $A \otimes_{B} A$ is a commutative $A-B$ module.

Theorem 1 (see [35], Theorem 2.1). Let $A$ and $B$ be Banach algebras such that $A$ is a $B$-bimodule, and suppose that $A \otimes_{B} A$ is a commutative $A-B$ module. Then the following statements are equivalent:

1) $A$ is $B$-module amenable and $A / J_{B}$ has a b.a.i.;

2) A has a B-module approximate diagonal.

(We omit a third part of this equivalence which is not necessary for our purposes.)

Note that this theorem does not establish equivalent conditions for module amenability per se. This is in contrast to the intrinsic characterization of amenability of a Banach algebra which was proved in [20, p. 264], and used by the authors in [26] to show that amenability was hereditary for certain spaces of functions taking their values in (possibly varying) Banach algebras. 
It is easy to check the following: 1$)$ if $A$ is commutative as a $B$-module, then $A \otimes_{B} A$ is commutative and $J_{B}=\{0\}$; and 2) if $A$ has a b.a.i. and $A \otimes_{B} A$ is commutative as a $B$-module, then so is $A / J_{B}$.

Henceforth, unless otherwise noted, we will let $X$ be a compact Hausdorff space, and $\rho: \mathcal{A}=\dot{\bigcup}\left\{A_{x}: x \in X\right\} \rightarrow X$ and $\pi: \mathcal{B}=\left\{B_{x}: x \in X\right\} \rightarrow X$ be bundles of Banach algebras such that the following standard assumptions hold:

S1) each $A_{x}$ is a $B_{x}$-bimodule satisfying condition (1);

S2) each $A_{x}$ has a $B_{x}$-module approximate diagonal $\left\{u_{\lambda_{x}}: \lambda_{x} \in \Lambda_{x}\right\}$, with $\left\|u_{\lambda_{x}}\right\| \leq p_{x}$, and the $B_{x}$-module approximate diagonals are uniformly bounded with $\sup _{x} p_{x}<p<\infty$;

S3) each $A_{x} \otimes_{B_{x}} A_{x}$ is a commutative $B_{x}$-module;

S4) $\rho$ is a bundle of bimodules over $\pi$.

Then, because the operations are pointwise-defined, $\Gamma(\rho)$ is a $\Gamma(\pi)$-bimodule which also satisfies condition (1). We will denote $\mathfrak{A}:=\Gamma(\rho)$ and $\mathfrak{B}:=\Gamma(\pi)$. Note that each of $\mathfrak{A}$ and $\mathfrak{B}$ is also a $C(X)$-module, and that each of them is necessarily commutative over $C(X)$. Denote by $K_{C(X)}$ and $K_{\mathfrak{B}}$ the $C(X)$ and $\mathfrak{B}$-balanced kernels, respectively, in $\mathfrak{A} \widehat{\otimes} \mathfrak{A}$. Denote by $J_{\mathfrak{B}} \subset \mathfrak{A}$ the closure of $\omega\left(K_{\mathfrak{B}}\right)$, where $\omega: \mathfrak{A} \widehat{\otimes} \mathfrak{A} \rightarrow \mathfrak{A}$ is the multiplication map in Figure 3. We will also generally assume that

S5) $K_{C(X)} \subset K_{\mathfrak{B}}(\subset \mathfrak{A} \widehat{\otimes} \mathfrak{A})$.

Before we prove a series of results leading up to the proof of Theorem 2, we note that the condition S5) that $K_{C(X)} \subset K_{\mathfrak{B}}$ may frequently hold.

Lemma 1. Let $\mathfrak{A}$ and $\mathfrak{B}$ satisfy $\mathbf{S 1})-\mathbf{S} 4)$ of the standard assumptions. If $\mathfrak{B}$ has an approximate identity $\left\{\beta_{i}\right\}$ for $\mathfrak{A}$, then $K_{C(X)} \subset K_{\mathfrak{B}}$, and $K_{C(X)}$ is a $\mathfrak{B}$-bimodule. Especially, this is true when $\pi$ is a bundle with identity and $\rho$ is a unital $\mathfrak{B}$-module.

Proof. Let $\left\{\beta_{i}\right\} \subset \mathfrak{B}$ be an approximate identity for $\mathfrak{A}$, and let $\sigma, \tau \in \mathfrak{A}$, $f \in C(X)$, and $\beta \in \mathfrak{B}$. Let $t=\sigma f \otimes \tau-\sigma \otimes f \tau \in K_{C(X)}$, and set

$$
t_{i}=(\sigma f) \beta_{i} \otimes \tau-\sigma \otimes \beta_{i}(\tau f)=\sigma\left(f \beta_{i}\right) \otimes \tau-\sigma \otimes\left(f \beta_{i}\right) \tau \in K_{\mathfrak{B}} .
$$

We then have

$$
\begin{aligned}
\left\|t_{i}-t\right\| & \leq\left\|f\left(\sigma \beta_{i}-\sigma\right) \otimes \tau\right\|+\left\|\sigma \otimes\left(\beta_{i} \tau-\tau\right) f\right\| \\
& \leq\|f\|\left(\left\|\sigma \beta_{i}-\sigma\right\|\|\tau\|+\left\|\beta_{i} \tau-\tau\right\|\|\sigma\|\right) \\
& \rightarrow 0 .
\end{aligned}
$$

Since the $t_{i}$ are in the closed set $K_{\mathfrak{B}}$, so also is $t \in K_{\mathfrak{B}}$. It is then evident that finite sums $\sum_{k=1}^{n}\left(\sigma_{k} f_{k} \otimes \tau_{k}-\sigma_{k} \otimes f_{k} \tau_{k}\right) \in K_{C(X)}$ are also in $K_{\mathfrak{B}}$, and since sums of this form are dense in $K_{C(X)}$, it follows that $K_{C(X)} \subset K_{\mathfrak{B}}$. 
Note also that

$$
\begin{aligned}
\beta t & =\beta[(\sigma f) \otimes \tau-\sigma \otimes(\tau f)] \\
& =(\beta \sigma) f \otimes \tau-(\beta \sigma) \otimes f \in K_{C(X)},
\end{aligned}
$$

and similarly for right actions, so that $K_{C(X)}$ is a $\mathfrak{B}$-bimodule.

We leave until later a demonstration of a condition sufficient to ensure that $\mathfrak{B}$ has a bounded approximate identity for $\mathfrak{A}$.

Lemma 2. Let $\mathfrak{A}$ and $\mathfrak{B}$ satisfy $\mathbf{S 1}$ )-S5) of the standard assumptions, and let $J_{\mathfrak{B}} \subset \mathfrak{A}$ be as defined. Then $\mathfrak{A} \otimes_{C(X)} \mathfrak{A}, \mathfrak{A} \otimes_{\mathfrak{B}} \mathfrak{A}$, and $\mathfrak{A} / J_{\mathfrak{B}}$ are all $C(X)$-bimodules. Moreover, the induced map $\widetilde{\omega}: \mathfrak{A} \otimes_{\mathfrak{B}} \mathfrak{A} \rightarrow \mathfrak{A} / J_{\mathfrak{B}}$ of Figure 3 is a $C(X)$-module homomorphism.

Proof. Clearly, $\mathfrak{A} \widehat{\otimes} \mathfrak{A}$, and hence $\mathfrak{A} \otimes_{C(X)} \mathfrak{A}$ are $C(X)$-modules. Moreover, for $\sigma \beta \otimes \tau-\sigma \otimes \beta \tau \in K_{\mathfrak{B}}$ and $f \in C(X)$, we have

$$
f(\sigma \beta \otimes \tau-\sigma \otimes \beta \tau)=(f \sigma) \beta \otimes \tau-(f \sigma) \otimes \beta \tau \in K_{\mathfrak{B}},
$$

and similarly for right action by $f$. Therefore, $K_{\mathfrak{B}}$ is a $C(X)$-module, and hence $\mathfrak{A} \otimes_{\mathfrak{B}} \mathfrak{A}$ is a $C(X)$-module.

To show that $J_{\mathfrak{B}}$ is a $C(X)$-module, let $f \in C(X)$, and let $\beta \in \mathfrak{B}$ and $\sigma, \tau \in \mathfrak{A}$. Then by linearity and continuity it suffices to observe that for $z=\sigma \beta \otimes \tau-\sigma \otimes \beta \tau \in K_{\mathfrak{B}}$ (because the multiplication is pointwise, and $f$ is $\mathbb{K}$-valued) we have

$$
\begin{aligned}
f \widetilde{\omega}(z) & =f(\sigma \beta) \tau-f \sigma(\beta \tau)=(f(\sigma \beta)) \tau-(f \sigma)(\beta \tau) \\
& =\widetilde{\omega}(f(\sigma \beta) \otimes \tau-(f \sigma) \otimes(\beta \tau)) \\
& =\widetilde{\omega}(f z) \\
& \in \widetilde{\omega}\left(K_{\mathfrak{B}}\right) \subset J_{\mathfrak{B}}(\subset \mathfrak{A}),
\end{aligned}
$$

and so $\mathfrak{A} / J_{\mathfrak{B}}$ is also a $C(X)$-module (and $\widetilde{\omega}$ is a $C(X)$-module homomorphism).

Corollary 1. Let $\mathfrak{A}$ and $\mathfrak{B}$ satisfy $\mathbf{S 1 )}$-S5) of the standard assumptions. We can identify $J_{\mathfrak{B}} \subset \mathfrak{A}$ with the section space $\Gamma(\eta)$ of the bundle $\eta: \dot{\bigcup} J_{B_{x}} \rightarrow X$, so that $\mathfrak{A} / J_{\mathfrak{B}}$ can be identified with the section space of a bundle $\chi: \dot{\bigcup} A_{x} / J_{B_{x}} \rightarrow X$, where for $\sigma \in \mathfrak{A}$ we have $\sigma+J_{\mathfrak{B}} \leftrightarrow(x \mapsto$ $\left.\sigma(x)+J_{B_{x}}\right) \in \mathfrak{N}=\Gamma(\chi)$.

Proof. We note that $J_{\mathfrak{B}}$ is a $C(X)$-submodule (necessarily $C(X)$-locally convex) of $\mathfrak{A}$, with fibers (isometric to) $J_{B_{x}}$. Thus, from I) of Section 2, the $C(X)$-module $\mathfrak{A} / J_{\mathfrak{B}}$ is also $C(X)$-locally convex, and can be identified with the section space $\Gamma(\chi)$ of the bundle $\chi: \dot{\bigcup} A_{x} / J_{B_{x}} \rightarrow X$. Then, again by I) of Section 2, the correspondence between $\mathfrak{A} / J_{\mathfrak{B}}$ and $\Gamma(\chi)$ is as claimed. 
Lemma 3. Let $\mathfrak{A}$ and $\mathfrak{B}$ satisfy $\mathbf{S 1 )}-\mathbf{S} 5$ ) of the standard assumptions. Then $\mathfrak{A} \otimes_{\mathfrak{B}} \mathfrak{A}$ is a $C(X)$-locally convex module. In particular, there is a bundle $\rho \otimes_{\pi} \rho: \mathcal{M}=\dot{\bigcup} A_{x} \otimes_{B_{x}} A_{x} \rightarrow X$ such that $\mathfrak{M}:=\Gamma\left(\rho \otimes_{\pi} \rho\right)$ is isometrically $C(X)$-isomorphic to $\mathfrak{A} \otimes_{\mathfrak{B}} \mathfrak{A}$. The correspondence is given by $\sigma \otimes_{\mathfrak{B}} \tau:=\sigma \otimes \tau+K_{\mathfrak{B}} \leftrightarrow \sigma \odot_{\mathfrak{B}} \tau$, where

$$
\left(\sigma \odot_{\mathfrak{B}} \tau\right)(x)=\sigma(x) \otimes_{B_{x}} \tau(x)=\sigma(x) \otimes \tau(x)+K_{B_{x}} .
$$

Proof. Since $K_{C(X)} \subset K_{\mathfrak{B}}$, it follows from ordinary algebraic considerations that there is a $C(X)$-isomorphism

$$
\mathfrak{A} \otimes_{\mathfrak{B}} \mathfrak{A}=(\mathfrak{A} \widehat{\otimes} \mathfrak{A}) / K_{\mathfrak{B}} \simeq\left[(\mathfrak{A} \widehat{\otimes} \mathfrak{A}) / K_{C(X)}\right] /\left[K_{\mathfrak{B}} / K_{C(X)}\right] .
$$

That the relation $\simeq$ is also an isometry follows because all the maps involved in proving the algebraic identity are norm-decreasing.

Having established the $C(X)$-isometric isomorphism between the two sides of $\simeq$, now consider the right-hand side. Since $\mathfrak{A}$ is $C(X)$-locally convex, it follows from [34, Theorem 1.2 and Proposition 1.5] that $(\mathfrak{A} \widehat{\otimes} \mathfrak{A}) / K_{C(X)}=$ $\mathfrak{A} \otimes_{C(X)} \mathfrak{A}$ can be identified with the section space $\Gamma\left(\rho \otimes_{C(X)} \rho\right)$ of a bundle with fibers $A_{x} \widehat{\otimes} A_{x}$, and the image of $\sigma \otimes \tau+K_{C(X)} \in(\mathfrak{A} \widehat{\otimes} \mathfrak{A}) / K_{C(X)}$ is $\sigma \odot \tau \in \Gamma\left(\rho \otimes_{C(X)} \rho\right)$, with $(\sigma \odot \tau)(x)=\sigma(x) \otimes \tau(x)$; in particular, $\mathfrak{A} \otimes_{C(X)} \mathfrak{A}$ is $C(X)$-locally convex. Then $K_{\mathfrak{B}} / K_{C(X)}$ is a $C(X)$-submodule of $(\mathfrak{A} \widehat{\otimes} \mathfrak{A}) / K_{C(X)}$, and therefore necessarily $C(X)$-locally convex. Under the identification above, an element $\sigma \beta \otimes \tau-\sigma \otimes \beta \tau+K_{C(X)} \in K_{\mathfrak{B}} / K_{C(X)}$ is mapped to $\sigma \beta \odot \tau-\sigma \odot \beta \tau \in \Gamma\left(\rho \otimes_{C(X)} \rho\right)$, with

$$
\begin{aligned}
(\sigma \beta \odot \tau-\sigma \odot \beta \tau)(x) & =\sigma(x) \beta(x) \otimes \tau(x)-\sigma(x) \otimes \beta(x) \tau(x) \\
& \in K_{B_{x}} \\
& \subset A_{x} \widehat{\otimes} A_{x} .
\end{aligned}
$$

The right-hand side of the isometric isomorphism is thus the quotient of two $C(X)$-locally convex modules (i.e., section spaces), and we now apply I) of Section 2 to the section spaces $\Gamma\left(\rho \otimes_{C(X)} \rho\right) \simeq(\mathfrak{A} \widehat{\otimes} \mathfrak{A}) / K_{C(X)}$ and $K_{\mathfrak{B}} / K_{C(X)}$ to obtain the result. In particular, we write $\sigma \otimes_{\mathfrak{B}} \tau \leftrightarrow \sigma \odot_{\mathfrak{B}} \tau$, where $\left(\sigma \odot_{\mathfrak{B}} \tau\right)(x)=\sigma(x) \otimes_{B_{x}} \tau(x)$, and the equation

$$
\begin{aligned}
\left\|\sum_{k} \sigma_{k} \otimes_{\mathfrak{B}} \tau_{k}\right\| & =\left\|\sum_{k} \sigma_{k} \odot_{\mathfrak{B}} \tau_{k}\right\| \\
& =\sup _{x}\left\|\sum_{k}\left(\sigma_{k} \odot_{\mathfrak{B}} \tau_{k}\right)(x)\right\| \\
& =\sup _{x}\left\|\sum_{k} \sigma_{k}(x) \otimes_{B_{x}} \tau_{k}(x)\right\|
\end{aligned}
$$

displays the isometry.

Corollary 2. Let $\mathfrak{A}$ and $\mathfrak{B}$ satisfy $\mathbf{S 1 )}-\mathbf{S 5}$ ) of the standard assumptions. If each $A_{x} \otimes_{B_{x}} A_{x}$ is commutative as a $B_{x}$-module, then $\mathfrak{A} \otimes_{\mathfrak{B}} \mathfrak{A}$ is commutative as a $\mathfrak{B}$-module. 
Proof. Let $\Omega: \mathfrak{A} \otimes_{\mathfrak{B}} \mathfrak{A} \rightarrow \mathfrak{M}=\Gamma\left(\rho \otimes_{\pi} \rho\right)$ be the isometric isomorphism established in Lemma 3. Let $\sigma, \tau \in \mathfrak{A}, \beta \in \mathfrak{B}$. For $x \in X$, we have

$$
\begin{aligned}
\Omega\left(\beta\left(\sigma \otimes_{\mathfrak{B}} \tau\right)\right)(x) & =\Omega\left((\beta \sigma) \otimes_{\mathfrak{B}} \tau\right)(x)=(\beta \sigma)(x) \otimes_{B_{x}} \tau(x) \\
& =\beta(x)\left(\sigma(x) \otimes_{B_{x}} \tau(x)\right) \\
& =\left(\sigma(x) \otimes_{B_{x}} \tau(x)\right) \beta(x) \\
& =\sigma(x) \otimes_{B_{x}}(\tau(x) \beta(x)) \\
& =\Omega\left(\sigma \otimes_{\mathfrak{B}} \tau \beta\right)(x) \\
& =\Omega\left(\left(\sigma \otimes_{\mathfrak{B}} \tau\right) \beta\right)(x),
\end{aligned}
$$

i.e., $\Omega\left(\beta\left(\sigma \otimes_{\mathfrak{B}} \tau\right)\right)=\Omega\left(\left(\sigma \otimes_{\mathfrak{B}} \tau\right) \beta\right)$, so that $\beta\left(\sigma \otimes_{\mathfrak{B}} \tau\right)=\left(\sigma \otimes_{\mathfrak{B}} \tau\right) \beta$, and thus $\mathfrak{A} \otimes_{\mathfrak{B}} \mathfrak{A}$ is commutative.

In the following, because of the identification of $\mathfrak{A} \otimes_{\mathfrak{B}} \mathfrak{A}$ and $\mathfrak{M}:=\Gamma\left(\rho \otimes_{\pi} \rho\right)$, we will simply work with $\mathfrak{M}$ without making the isometric isomorphism explicit via a given map. The same will be true for $\mathfrak{N} \simeq \mathfrak{A} / J_{\mathfrak{B}}$. In doing this, we will be transferring the difficult task of finding norms in quotient spaces to the perhaps easier task of finding pointwise norms (admittedly, of quotients) in section spaces.

Let $\mathfrak{A}$ and $\mathfrak{B}$ satisfy $\mathbf{S 1}$ )-S5) of the standard assumptions, and let $\left\{u_{\lambda_{x}}: \lambda_{x} \in \Lambda_{x}\right\} \subset A_{x} \otimes_{B_{x}} A_{x}$ be the $B_{x}$-module approximate diagonal for $A_{x}$. For $\lambda \in \Lambda=\prod_{x \in X} \Lambda_{x}$, write $\lambda(x)=\lambda_{x}$ and order $\Lambda$ pointwise, i.e., $\lambda^{\prime} \geq \lambda$ if and only if $\lambda^{\prime}(x) \geq \lambda(x)$ for each $x \in X$. Given $\lambda \in \Lambda$, for each $x \in X$, choose and fix $\nu_{\lambda(x)} \in \mathfrak{M}$ such that $\nu_{\lambda(x)}(x)=u_{\lambda_{x}}$ and such that $\left\|\nu_{\lambda(x)}\right\|=\left\|u_{\lambda_{x}}\right\|<p$ (where $p$ is the uniform bound on the $\left.\left\|u_{\lambda(x)}\right\|\right)$ ); the existence of such a $\nu_{\lambda(x)}$ is guaranteed by [30, Proposition 1.1]. For given $\lambda \in \Lambda, x \in X$ and $\sigma \in \mathfrak{A}$, and letting $\widetilde{\omega_{x}}: A_{x} \otimes_{B_{x}} A_{x} \rightarrow A_{x} / J_{B_{x}}$ be the map $\widetilde{\omega}$ of Figure 3 applied to $A_{x} \otimes_{B_{x}} A_{x}$, note that we have

$$
\begin{aligned}
\left\|\nu_{\lambda(x)}(x) \sigma(x)-\sigma(x) \nu_{\lambda(x)}(x)+K_{B_{x}}\right\| & =\left\|u_{\lambda(x)} \sigma(x)-\sigma(x) u_{\lambda(x)}+K_{B_{x}}\right\| \\
& \rightarrow 0,
\end{aligned}
$$

and that both

$$
\left\|\sigma(x) \widetilde{\omega}\left(\nu_{\lambda(x)}(x)\right)-\sigma(x)+J_{B_{x}}\right\|=\left\|\sigma(x) \widetilde{\omega_{x}}\left(u_{\lambda(x)}\right)-\sigma(x)+J_{B_{x}}\right\| \rightarrow 0,
$$

and

$$
\left\|\widetilde{\omega}\left(\nu_{\lambda(x)}(x)\right) \sigma(x)-\sigma(x)+J_{B_{x}}\right\|=\left\|\widetilde{\omega_{x}}\left(u_{\lambda(x)}\right) \sigma(x)-\sigma(x)+J_{B_{x}}\right\| \rightarrow 0,
$$

where the norms are taken in the appropriate spaces.

We will construct a $\mathfrak{B}$-module approximate diagonal for $\mathfrak{A}$ of norm less than $p$.

Lemma 4. Let $\mathfrak{A}$ and $\mathfrak{B}$ satisfy $\mathbf{S 1 ) - S 5 )}$ of the standard assumptions, and let $F=\left\{\sigma_{1}, \ldots, \sigma_{n}\right\} \subset \mathfrak{A}$ and $m \in \mathbb{N}$ be given. Suppose that 
$\rho \otimes_{\pi} \rho: \mathcal{M}=\dot{\bigcup} A_{x} \otimes_{B_{x}} A_{x} \rightarrow X$ is the tensor product bundle. Then there exists $\xi=\xi(F, m) \in \mathfrak{M}=\Gamma\left(\rho \otimes_{\pi} \rho\right) \simeq \mathfrak{A} \otimes_{\mathfrak{B}} \mathfrak{A}$ such that

1) $\|\xi\|<p$

and, for each $\sigma \in F$,

2) $\|\xi \sigma-\sigma \xi\|=\sup _{x}\left\|\xi(x) \sigma(x)-\sigma(x) \xi(x)+K_{B_{x}}\right\|<1 / m($ in $\mathfrak{M})$,

3) $\|\widetilde{\omega}(\xi) \sigma-\sigma\|=\sup _{x}\left\|\widetilde{\omega}_{x}(\xi) \sigma(x)-\sigma(x)+K_{J_{B}}\right\|<1 / m$ and $\|\sigma \widetilde{\omega}(\xi)-\sigma\|=\sup _{x}\left\|\sigma(x) \widetilde{\omega}_{x}(\xi)-\sigma(x)+J_{B_{x}}\right\|<1 / m$ (both in $\mathfrak{N}$ ).

Here, if $\xi=\sum_{k} \tau_{k} \odot_{\mathfrak{B}} \tau_{k}^{\prime}$, the multiplication is given by, say,

$$
(\xi \sigma)(x)=\sum_{k}\left(\tau_{k} \odot_{B_{x}} \tau_{k}^{\prime}\right)(x) \sigma(x)=\sum_{k} \tau(x) \otimes_{B_{x}}\left(\tau^{\prime}(x) \sigma(x)\right) .
$$

Proof. Let $m \in \mathbb{N}$ and $F \subset \mathfrak{A}$ be given. As above, write $u_{\lambda_{x}}=\nu_{\lambda(x)}(x)$ for $\nu_{\lambda(x)} \in \mathfrak{M}$ with $\nu_{\lambda(x)}(x)=u_{\lambda_{x}}$ and $\left\|\nu_{\lambda(x)}\right\|=\left\|u_{\lambda_{x}}\right\| \leq p_{x}<p$. Let $\sigma_{k} \in F, x \in X$, and $m \in \mathbb{N}$ be given. Since $\left\{u_{\lambda_{x}}\right\}=\left\{\nu_{\lambda(x)}(x)\right\}$ is a $B_{x^{-}}$ module approximate diagonal for $A_{x}$, we can define $\lambda_{m, k} \in \Lambda$ by choosing $\lambda_{m, k}(x) \in \Lambda_{x}$ such that if $\lambda(x) \geq \lambda_{m, k}(x)$ each of

$$
\begin{gathered}
\left\|\nu_{\lambda_{m, k}(x)}(x) \sigma_{k}(x)-\sigma_{k}(x) \nu_{\lambda_{m, k}(x)}(x)+K_{B_{x}}\right\|<1 / m\left(\text { in } A_{x} \otimes_{B_{x}} A_{x}\right), \\
\left.\left\|\widetilde{\omega}\left(\nu_{\lambda_{m, k}(x)}(x)\right) \sigma_{k}(x)-\sigma_{k}(x)+J_{B_{x}}\right\|<1 / m \text { (in } A_{x} / J_{B_{x}}\right),
\end{gathered}
$$

and

$$
\left.\left\|\sigma_{k}(x) \widetilde{\omega}\left(\nu_{\lambda_{m, k}(x)}\right)(x)-\sigma_{k}(x)+J_{B_{x}}\right\|<1 / m \text { (in } A_{x} / J_{B_{x}}\right) .
$$

Then if $\lambda_{m} \in \Lambda$ is such that $\lambda_{m} \geq \max \left\{\lambda_{m, k}: k=1, \ldots, n\right\}$, then the above inequalities hold for each $\sigma \in F$ and $x \in X$.

From the upper semicontinuity of the norm functions in $\mathfrak{M}$ and $\mathfrak{N}$, we can then choose a neighborhood $V_{x}(F, m)$ of $x$ such that if $y \in V_{x}(F, m)$, then $\left\|\nu_{\lambda_{m}(x)}(y) \sigma(y)-\sigma(y) \nu_{\lambda_{m}(x)}(y)+K_{B_{y}}\right\|<1 / m$ (in $A_{y} \otimes_{B_{y}} A_{y}$ ), and similarly for ( ††) and ( $\dagger \dagger \dagger)$, for all $\sigma \in F$.

Now, $X$ is compact, so we can choose $\left\{x_{j}: j=1, \ldots, s\right\} \subset X$ such that $\left\{V_{j}\right\}=\left\{V_{x_{j}}(F, m), j=1, \ldots, s\right\}$ also covers $X$. Let $\left\{f_{j}: j=1, \ldots, s\right\}$ be a partition of unity subordinate to the $V_{j}$, and define $\xi=\xi(F, m) \in \mathfrak{M}$ by $\xi=\sum_{j=1}^{s} f_{j} \nu_{\lambda_{m}\left(x_{j}\right)}$. Because $\left\|\nu_{\lambda_{m}}\right\|<p$, we then have, for $y \in X$,

$$
\begin{aligned}
\|\xi(y)\| & =\left\|\sum_{j=1}^{s} f_{j}(y) \nu_{\lambda\left(x_{j}\right)}(y)\right\| \\
& \leq \sum_{j \text { s.t. } y \in V_{j}} f_{j}(y)\left\|\nu_{\lambda\left(x_{j}\right)}(y)\right\|<\sum_{j \text { s.t. } y \in V_{j}} f_{j}(y) p \\
& \leq p,
\end{aligned}
$$

so that $\|\xi\| \leq p$. 
Moreover, for $y \in X$ and $\sigma \in F$, setting $p^{\prime}=\|\xi(y) \sigma(y)-\sigma(y) \xi(y)\|$, we have

$$
\begin{aligned}
p^{\prime} & =\left\|\sum_{j \text { s.t. } y \in V_{j}} f_{j}(y)\left[\nu_{\lambda_{m}\left(x_{j}\right)}(y) \sigma(y)-\sigma(y) \nu_{\lambda_{m}\left(x_{j}\right)}(y)\right]+K_{B_{y}}\right\| \\
& \leq \sum_{j \text { s.t. } y \in V_{j}} f_{j}(y)\left\|\nu_{\lambda_{m}\left(x_{j}\right)}(y) \sigma(y)-\sigma(y) \nu_{\lambda_{m}\left(x_{j}\right)}(y)+K_{B_{y}}\right\| \\
& <\sum_{j \text { s.t. } y \in V_{j}} f_{j}(y) \cdot(1 / m) \leq 1 / m,
\end{aligned}
$$

so that $\|\xi \sigma-\sigma \xi\|<1 / m$ (in $\mathfrak{M}$ ) for each $\sigma \in F$.

Similarly, both $\|\widetilde{\omega}(\xi) \sigma-\sigma\|<1 / m$ and $\|\sigma \widetilde{\omega}(\xi)-\sigma\|<1 / m$ (in $\mathfrak{N}$ ) for each $\sigma \in F$.

Corollary 3. Let $\mathfrak{A}$ and $\mathfrak{B}$ satisfy $\mathbf{S 1 ) - S 5 )}$ of the standard assumptions. Then $\mathfrak{A}$ has a $\mathfrak{B}$-module approximate diagonal (of norm $p$ ).

Proof. We adapt the proof of Proposition 1.2 of [13].

Set $\Psi=\{(F, m): F \subset \mathfrak{A}$ is finite, $m \in \mathbb{N}\}$, and order $\Psi$ by $\left(F^{\prime}, m^{\prime}\right)>(F, m)$ if $F^{\prime} \supset F$ and $m^{\prime}>m$. Then, by Lemma 4, for each pair $(F, m) \in \Psi$ there exists $\xi \in \mathfrak{M}$, with $\|\xi\| \leq p$, such that all of $\|\xi \sigma-\sigma \xi\|<1 / m,\|\widetilde{\omega}(\xi) \sigma-\sigma\|<1 / m$, and $\|\sigma \widetilde{\omega}(\xi)-\sigma\|<1 / m$ hold for all $\sigma \in F$. In particular, for a given $\sigma_{0} \in \mathfrak{A}$, and $m_{0} \in \mathbb{N}$, there exists $\left(F_{0}, m_{0}\right) \in \Psi$, with $\sigma_{0} \in F_{0}$, such that if $\left(F^{\prime}, m^{\prime}\right)>\left(F_{0}, m_{0}\right)$ then $\left\|\xi^{\prime} \sigma_{0}-\sigma_{0} \xi^{\prime}\right\|, \quad\left\|\widetilde{\omega}\left(\xi^{\prime}\right) \sigma_{0}-\sigma_{0}\right\|$, and $\left\|\sigma_{0} \widetilde{\omega}\left(\xi^{\prime}\right)-\sigma_{0}\right\|$ are all less than $1 / m^{\prime}<1 / m_{0}$, where $\xi^{\prime}=\xi^{\prime}\left(F^{\prime}, m^{\prime}\right)$ is constructed as in the proof of Lemma 4. Thus, $\{\xi=\xi(F, m): F \subset \mathfrak{A}, m \in \mathbb{N}\}$ is a $\mathfrak{B}$-module approximate diagonal for $\mathfrak{A}$.

Theorem 2. Let $\mathfrak{A}$ and $\mathfrak{B}$ satisfy $\mathbf{S 1 ) - S 5 )}$ of the standard assumptions. Then $\mathfrak{A}$ is $\mathfrak{B}$-module amenable and $\mathfrak{A} / J_{\mathfrak{B}}$ has a bounded approximate identity.

Proof. By Corollary $3, \mathfrak{A}$ has a $\mathfrak{B}$-module approximate diagonal, and so by Theorem 1 it is $\mathfrak{B}$-module amenable (and $\mathfrak{A} / J_{\mathfrak{B}}$ has a bounded approximate identity).

The sequence of results ending in Theorem 2 shows that if we are given conditions S1)-S5), then a $\mathfrak{B}$-module approximate diagonal for $\mathfrak{A}$ (and hence the $\mathfrak{B}$-module amenability of $\mathfrak{A}$ and the existence of a bounded approximate identity in $\left.\mathfrak{A} / J_{\mathfrak{B}}\right)$ is inherited from the fibers $A_{x}$ and $B_{x}$. What of the converse? Does the commutativity of $\mathfrak{A} \otimes_{\mathfrak{B}} \mathfrak{A}$ as a $\mathfrak{B}$-module and existence of a $\mathfrak{B}$-module approximate diagonal for $\mathfrak{A}$ (and hence the $\mathfrak{B}$-module amenability of $\mathfrak{A}$ and the existence of a bounded approximate identity in $\left.\mathfrak{A} / J_{\mathfrak{B}}\right)$ imply the same things for the fibers $A_{x}$ and $B_{x}$ ? The answer is yes, as can easily be seen. For, identify $\mathfrak{A} \otimes_{\mathfrak{B}} \mathfrak{A}$ as the section space $\Gamma\left(\rho \otimes_{\pi} \rho\right)$ 
whose existence is established in Lemma 3. If this section space is a commutative $\mathfrak{B}$-module, then the proof of Lemma 2 shows that each $A_{x} \otimes_{B_{x}} A_{x}$ is a commutative $B_{x}$-module. Moreover, if $\left\{\nu_{\lambda}\right\} \subset \mathfrak{A} \otimes_{\mathfrak{B}} \mathfrak{A}$ is a $\mathfrak{B}$-module approximate diagonal for $\mathfrak{A}$, then $\left\{\phi_{x}\left(\nu_{\lambda}\right)\right\}$ is a $B_{x}$-module approximate diagonal for $A_{x}$, where $\phi_{x}: \Gamma\left(\otimes_{\pi} \rho\right) \simeq \mathfrak{A} \otimes_{\mathfrak{B}} \mathfrak{A} \rightarrow A_{x} \otimes_{B_{x}} A_{x}$ is the evaluation map. Since $\left\|\phi_{x}\right\| \leq 1$, these $B_{x}$-module approximate diagonals are uniformly bounded. A similar argument can be applied to $\left\{\widetilde{\omega}\left(\nu_{\lambda}\right)\right\} \subset \mathfrak{A} / J_{\mathfrak{B}}$ to obtain uniformly bounded approximate identities in the spaces $A_{x} / J_{B_{x}}$.

We can also modify some assumptions about the $A_{x}$ and $B_{x}$.

Proposition 1. Let $A$ and $B$ be Banach algebras such that $A$ is a commutative $B$-module satisfying conditions (1) and (2) (on $A$ and $B$ ), and hence also the condition that $a\left(b a^{\prime}\right)=(a b) a^{\prime}$, for $a, a^{\prime} \in A, b \in B$, so that $A$ is a commutative $A-B$ module. If $A$ is $B$-module amenable, then it has a bounded approximate identity.

Proof. This is Proposition 2.2 of [2], taking into account the remarks in $[3]$.

Lemma 5. If $A$ is a commutative $A-B$ module which is also $B$-module amenable, then it has a $B$-module approximate diagonal.

Proof. Let $\omega: A \widehat{\otimes} A \rightarrow A$ be the multiplication map, $a, a^{\prime} \in A, b \in B$, and let $K_{B} \subset A \widehat{\otimes} A$ be the $B$-balanced kernel in $A \widehat{\otimes} A$. Then $a b \otimes a^{\prime}-a \otimes b a^{\prime} \in K_{B}$, and as noted in the statement of Proposition 1, we have $\omega\left(a b \otimes a^{\prime}-a \otimes b a^{\prime}\right)=(a b) a^{\prime}-a\left(b a^{\prime}\right)=0$. Hence $\omega\left(K_{B}\right)=0$, and $A / J_{B}=A$, which by the preceding proposition has a bounded approximate identity. Thus, by Theorem $1, A$ has a $B$-module approximate diagonal.

Corollary 4. Suppose that $\mathfrak{A}$ and $\mathfrak{B}$ satisfy $\mathbf{S 1 ) - S 5 )}$ of the standard assumptions and that each pair $A_{x}, B_{x}$ satisfies the additional condition noted in the hypothesis of Proposition 1. Then $\mathfrak{A}$ has a bounded approximate identity.

Proof. It can be easily checked that $\mathfrak{A}$ and $\mathfrak{B}$ also satisfy the condition of Theorem 1. Hence, as in the proof of Lemma $5, J_{\mathfrak{B}}=0$, and so $\mathfrak{A} / J_{\mathfrak{B}}=\mathfrak{A}$ has a bounded approximate identity.

The standard examples for the heritability of amenability, in its usual sense, are the following: 1$)$ if $A$ is amenable, then $C(X, A)$ is amenable; and 2) if $\left\{A_{x}: x \in X\right\}$ is a collection of uniformly amenable algebras (see [26, Definition 2.6]), then $c_{0}\left(X, \dot{\bigcup}\left\{A_{x}: x \in X\right\}\right)$, the $c_{0}$-sum of the $A_{x}$, is amenable. The following is the analogue of those examples for module amenability.

Corollary 5. Let $B$ be a fixed Banach algebra. Suppose that $\rho: \mathcal{A}=$ $\dot{\bigcup}\left\{A_{x}: x \in X\right\} \rightarrow X$ is a bundle of Banach algebras such that each 
$A_{x}$ is a B-bimodule. Suppose further that the $A_{x}$ have uniformly bounded $B$-module approximate diagonals, and that $\mathfrak{A}=\Gamma(\rho)$ is a B-bimodule (under the operations $(b \sigma)(x)=b \sigma(x)$ and $(\sigma b)(x)=\sigma(x) b)$ and that $K_{C(X)} \subset K_{B}$. Then $\mathfrak{A}$ has a $B$-module approximate diagonal. As consequences, then

$1)$ if the fixed algebra $A$ has a $B$-module approximate diagonal, then so does $\mathfrak{A}=C(X, A) ;$ and

2) $c_{0}\left(X, \dot{\bigcup}\left\{A_{x}: x \in X\right\}\right)$ has a $B$-module approximate diagonal.

Proof. If $\mathfrak{A}$ is a $B$-module, then it is also a $C(X, B)$-module. For, let $f \in C(X), b \in B$, and $\sigma \in \mathfrak{A}$. Define $f * b \in C(X, B)$ by $(f * b)(x)=f(x) b$; we then have $(f * b) \sigma \in \mathfrak{A}$. But $C(X, B)$ is the closed span in the uniform norm of elements of the form $f * b$, so that if $\beta \in C(X, B)$ it follows by density that $\beta \sigma \in \mathfrak{A}$ also. Note that $B$ is isometrically a subspace of $C(X, B)$ under the correspondence $b \leftrightarrow \mathbf{1} * b$, where $\mathbf{1}$ is the constant function on $X$ with value 1 . From this, it is clear that $K_{B}$, the closed span in $\mathfrak{A} \widehat{\otimes} \mathfrak{A}$ of elements of the form $\sigma b \otimes \tau-\sigma \otimes b \tau$, is a subset of $K_{C(X, B)}$. To show that $K_{C(X, B)} \subset K_{B}$, we simply repeat the density and linearity arguments used to show that $\mathfrak{A}$ is a $C(X, B)$-module.

We thus have

$$
\mathfrak{A} \otimes_{\mathfrak{B}} \mathfrak{A}=(\mathfrak{A} \widehat{\otimes} \mathfrak{A}) / K_{B}=(\mathfrak{A} \widehat{\otimes} \mathfrak{A}) / K_{C(X, B)}=\mathfrak{A} \otimes_{C(X, B)} \mathfrak{A} .
$$

If we now regard $C(X, B)$ as the section space of the trivial bundle $\rho: X \times B \rightarrow X$, the result follows from Theorem 2 and its preceding lemmas.

As for cases 1$)$ and 2$)$ they now follow by regarding $C(X, A)$ as the section space of the trivial bundle $\rho: X \times A \rightarrow X$, and $c_{0}\left(X, \dot{\bigcup}\left\{A_{x}: x \in X\right\}\right)$ as the section space of the spiky bundle $\rho_{0}: \dot{\bigcup}\left\{A_{x}: x \in X\right\} \rightarrow X$.

Note that the condition $K_{C(X)} \subset K_{B}$ will hold if $B$ has an approximate identity for $\mathfrak{A}$; this can be shown using the same ideas as in Lemma 1.

We had promised earlier to produce a condition sufficient for $\mathfrak{B}$ to have an approximate identity for $\mathfrak{A}$.

Proposition 2. Let $\rho: \mathcal{A}=\dot{\bigcup}\left\{A_{x}: x \in X\right\} \rightarrow X$ and $\pi: \mathcal{B}=\dot{\bigcup}\left\{B_{x}\right.$ : $x \in X\} \rightarrow X$ be bundles of Banach algebras such that each $A_{x}$ is a (left) $B_{x}$-module and such that $\mathfrak{A}:=\Gamma(\rho)$ is a module over $\mathfrak{B}:=\Gamma(\pi)$. Suppose further that the $B_{x}$ have uniformly bounded (left) approximate identities $\left\{b_{\lambda_{x}}: \lambda_{x} \in \Lambda_{x}\right\}$ for the $A_{x}$. Then $\mathfrak{B}$ has a bounded (left) approximate identity for $\mathfrak{A}$; conversely, if $\mathfrak{B}$ has a bounded (left) approximate identity for $\mathfrak{A}$, then the $B_{x}$ have uniformly bounded (left) approximate identities for the $A_{x}$.

Proof. Choose $p>\sup \left\{\left\|b_{\lambda_{x}}\right\|: \lambda_{x} \in \Lambda_{x}, x \in X\right\}$. Let $\Lambda=\prod\left\{\Lambda_{x}: x \in X\right\}$, and write $\lambda(x)=\lambda_{x}$. For each $\lambda(x)$, choose and fix $\beta_{\lambda(x)} \in \mathfrak{B}$ such that $\beta_{\lambda(x)}(x)=b_{\lambda_{x}}$ and such that $\left\|\beta_{\lambda(x)}\right\|=\left\|b_{\lambda_{x}}\right\|<p$. Now, as in the proof 
of Lemma 4 and Corollary 3, given a finite set $F \subset \mathfrak{A}$ and $m \in \mathbb{N}$, we can construct a net $\{\beta(F, m): F \subset \mathfrak{A}$ is finite and $m \in \mathbb{N}\} \subset \mathfrak{B}$ such that $\|\beta(F, m)\|<p$ and such that for each $\sigma \in \mathfrak{A},\|\beta(F, m) \sigma-\sigma\| \rightarrow 0$; again the net is ordered by $\left(F^{\prime}, m^{\prime}\right)>(F, m)$ if $F^{\prime} \supset F$ and $m^{\prime}>m$. As for the converse, note that the evaluation map $\phi_{x}: \mathfrak{A} \mapsto A_{x}$ is norm decreasing, so that if $\left\{\beta_{i}\right\}$ is an approximate identity bounded by $p$ for $\mathfrak{A}$, then for $x \in X$, $\left\{\beta_{i}(x)\right\}$ is an approximate identity bounded by $p$ for $A_{x}$.

Note that even if we do not assume that the approximate identities $\left\{b_{\lambda_{x}}\right\}$ above are uniformly bounded, we will still obtain an approximate identity in $\mathfrak{B}$ for $\mathfrak{A}$.

Recall that a (left) $A$-module $M$ is said to be essential provided that $M=A M$.

Proposition 3. Suppose that $\rho: \mathcal{M} \rightarrow X$ is a bundle of (left) Banach modules over $\pi: \mathcal{A} \rightarrow X$, and that each $M_{x}$ is essential as an $A_{x}$-module. Then $\mathfrak{M}=\Gamma(\rho)$ is essential as an $\mathfrak{A}=\Gamma(\pi)$-module. The converse also holds.

Proof. Consider $W:=\operatorname{span}\{\alpha \sigma: \alpha \in \mathfrak{A}, \sigma \in \mathfrak{M}\}$. By hypothesis, for each $x \in X$ the space $W_{x}=\operatorname{span}\{\alpha(x) \sigma(x): \sigma \in \mathfrak{A}, \sigma \in \mathfrak{M}\}$ is dense in $M_{x}$. It is evident that since both $\mathfrak{A}$ and $\mathfrak{M}$ are $C(X)$-modules, so is $W$. Then by the Stone-Weierstrass theorem for section spaces of bundles [17, Corollary 4.3], $W$ is dense in $\mathfrak{M}$; i.e., $\mathfrak{M}$ is essential as an $\mathfrak{A}$-module.

The converse holds by the same argument used in Proposition 2.

Recall that the Cohen Factorization Theorem (see, e.g., [13, Theorem 16.5]) states that if $M$ is an essential (left) $A$-module, and $A$ has a bounded approximate identity, then $M$ can be factored over $A$, i.e., if $z \in M$, there exists $a \in A$ and $y \in M$ such that $z=a y$. We then obtain the following corollary to Propositions 2 and 3, which provides a sort of uniform factorization for section spaces of module bundles over section spaces of algebra bundles.

Corollary 6. Suppose that $\rho: \mathcal{M} \rightarrow X$ is a bundle of (left) Banach modules over the algebra bundle $\pi: \mathcal{A} \rightarrow X$; set $\mathfrak{M}:=\Gamma(\rho)$ and $\mathfrak{A}:=\Gamma(\pi)$. If the fibers $A_{x}$ have uniformly bounded (left) approximate identities, and if each $M_{x}$ is essential as an $A_{x}$-module, then $\mathfrak{M}$ factors as an $\mathfrak{A}$-module. If $\sigma=\alpha \tau$ for $\alpha \in \mathfrak{A}$ and $\sigma, \tau \in \mathfrak{M}$, then this factorization of $\sigma$ determines a factorization of $\sigma(x)$ for each $x \in X$.

\section{Irreducibility of modules over algebra bundles}

It is well known that non-zero irreducible $C(X)$-modules are of dimension one. In [33] this result was demonstrated using the fact that $C(X)$ is essentially the section space of the trivial bundle $\pi: X \times \mathbb{K} \rightarrow X$. In this 
section we show that an analogous result is true for irreducible modules over the section spaces of certain algebra bundles. Recall that to say a (left) module $M \neq 0$ is irreducible over the algebra $A$ means that the only closed submodules of $M$ are $\{0\}$ and $M$ itself. Evidently, if $M$ is one-dimensional, it is irreducible.

Suppose that $\operatorname{dim} M \geq 2$, and that $M$ is irreducible. Then either $A M=\{0\}$ or $A M=M$. If $A M=\{0\}$, then every proper closed subspace of $M$ is a closed submodule, so that $M$ is not irreducible. Hence $A M=M$, and in terms of irreducibility we need only worry about essential modules of dimension $\geq 2$.

In this section, we assume that $M \neq 0$ is a (left) module over both $C(X)$ and $\mathfrak{A}$, where $\pi: \mathcal{A} \rightarrow X$ is an algebra bundle with fibers $A_{x}, \mathfrak{A}=\Gamma(\pi)$, that $\mathfrak{A}$ is itself essential as an $\mathfrak{A}$-module (so that $\mathfrak{A}^{2}=\mathfrak{A}$ ), and that $M$ is unital as a $C(X)$-module and essential as an $\mathfrak{A}$-module. (Especially, an $\mathfrak{A}$-module $M$ may also be taken to be a $C(X)$-module if $\mathfrak{A}$ has a identity $e$ of norm one and $M$ is unital over $\mathfrak{A}$ : if $f \in C(X)$ and $m \in M$, we set $f \cdot m:=(f e) \cdot m$, and then, aside from the easily checked arithmetic conditions, we have $\|f \cdot m\|=\|f e \cdot m\| \leq\|f e\|\|m\| \leq\|f\|\|m\|$.)

Lemma 6. Suppose that $\pi: \mathcal{A} \rightarrow X$ is an algebra bundle with fibers $A_{x}$. Set $\mathfrak{A}:=\Gamma(\pi)$. Let $M \neq 0$ be an $\mathfrak{A}$-irreducible (and hence $\mathfrak{A}$-essential) $C(X)$ - and $\mathfrak{A}$-module, which is unital as a $C(X)$-module, and suppose also that $\mathfrak{A}^{2}=\mathfrak{A}$. Then $M$ is $C(X)$-locally convex.

Proof. Let $U \subset X$ be a closed subset, and set

$$
I_{U}=\{f \in C(X): f(U)=0\} .
$$

Then it is easy to check that $I_{U} \mathfrak{A}$ is the $C(X)$-submodule of sections in $\mathfrak{A}$ which vanish on $U$. Now, let $U, V \subset X$ be closed, with $U \cup V=X$, so that $I_{U} I_{V}=0$. Then either $I_{U} \mathfrak{A} \cdot M$ or $I_{V} \mathfrak{A} \cdot M$ is 0 . For if both

$$
I_{U} \mathfrak{A} \cdot M=I_{V} \mathfrak{A} \cdot M=M,
$$

(which is the only other possibility, since $M$ is irreducible over $\mathfrak{A}$ ), then because $I_{U}$ and $I_{V}$ are scalar-valued and hence commute with $\mathfrak{A}$, we have

$$
M=I_{V} \mathfrak{A} \cdot M=I_{V} \mathfrak{A} \cdot\left(I_{U} \mathfrak{A} \cdot M\right)=I_{V} I_{U} \mathfrak{A}^{2} \cdot M=0,
$$

which is clearly impossible.

Suppose now that $M$ is not $C(X)$-locally convex. We may then find $f, g \in C(X)$ and $m \in M$ such that $f g=0$ and

$$
\|(f+g) m\| \neq \max \{\|f m\|,\|g m\|\} .
$$

Set $U=f^{-1}(\{0\})$ and $V=g^{-1}(\{0\})$; then $U \cup V=X$.

Assume that $\|(f+g) m\|>\max \{\|f m\|,\|g m\|\}$. Then $(f+g) m \neq 0$, so that at least one of $f m, g m \neq 0$, say $f m \neq 0$, i.e., $I_{U} \mathfrak{A} \cdot M=I_{U} M \neq 0$. 
Hence $I_{V} \mathfrak{A} \cdot M=0$, and so $g m=0$, and thus the strict inequality does not hold.

Similarly, suppose that $\|(f+g) m\|<\max \{\|f m\|,\|g m\|\}$. Then $f m \neq 0$, say, which forces $g m=0$, which again negates the inequality. Hence

$$
\|(f+g) m\|=\max \{\|f m\|,\|g m\|\},
$$

and $M$ is $C(X)$-locally convex.

Thus, from IV) of Section 2, there is a $C(X)$-isometric isomorphism $\sim: M \rightarrow \Gamma(\rho)$, where $\rho: \mathcal{F} \rightarrow X$ is the canonical bundle of $M$ (as a $C(X)$-module) with fibers $F_{x}=M / I_{x} M=M /\left(I_{x} \mathfrak{A} \cdot M\right)$. We will identify $M$ with $\Gamma(\rho)$, without making the isomorphic correspondence explicit. Note also that $\mathfrak{A}$ is by definition $C(X)$-locally convex, and that $\mathfrak{A} / I_{x} \mathfrak{A} \simeq A_{x}$. In the standard way, we can regard $F_{x}$ as an $\mathfrak{A} / I_{x} \mathfrak{A}$-module, and hence as an $A_{x}$-module. Thus, $\rho$ is a bundle of modules over a bundle of algebras, and action of $\mathfrak{A}$ on $M \simeq \Gamma(\rho)$ is given pointwise. Since $M \simeq \Gamma(\rho)$ is an essential $\mathfrak{A}$-module, each $F_{x}$ is also an essential $A_{x}$-module; see Proposition 3 . To the point for our purposes, note that since $M=\mathfrak{A} \cdot M$, we have $I_{x} M=I_{x} \mathfrak{A} \cdot M$, so that $I_{x} M$ is both an $\mathfrak{A}$ - and $C(X)$-submodule of $M$; hence by the $\mathfrak{A}$ irreducibility of $M$ we have for each $x \in X$ that $I_{x} M=\{0\}$ or $I_{x} M=M$. Thus $F_{x}=M / I_{x} M=M$ or $F_{x}=0$. Note that for any fixed $x \in X$, the bundle $\rho^{\prime}: \mathfrak{F}^{\prime} \rightarrow X$ whose fibers are $F_{y}^{\prime}=0$, if $y \neq x$, and $F_{x}^{\prime}=F_{x}=M / I_{x} M$ is what we called in II) of Section 2 a single-point bundle of modules over the algebra bundle $\mathcal{A}$, where the action of $\alpha \in \mathfrak{A}$ on $\sigma \in \Gamma\left(\rho^{\prime}\right)$ is given pointwise.

Suppose for the moment that there exist $x \neq y \in X$ such that $F_{x}=F_{y}=M$. Choose disjoint neighborhoods $U$ and $V$ of $x$ and $y$, respectively, and let $f, g \in C(X)$ be supported on $U$ and $V$ with $f(x)=g(y)=1$. Regarding $M$ as the space of sections $\Gamma(\rho)$, with fibers $M$ or $\{0\}$, it is then easy to check that $f M=f \mathfrak{A} \cdot M$ and $g M=g \mathfrak{A} \cdot M$ are distinct $\mathfrak{A}$-submodules of $M$, so that by the $\mathfrak{A}$-irreducibility of $M$ only one of them, say $f M$, is nontrivial, and hence isometrically isomorphic to $M$. It is then apparent that the action of $\mathfrak{A}$ on $M$ is given by $\alpha \cdot m=\alpha(x) \cdot m$. Thus, $\Gamma(\rho) \simeq M$ is the section space of a single-point bundle, and $M$ is irreducible as an $A_{x}$-module.

We have thus proved the following result.

Proposition 4. Assume that the conditions of Lemma 6 hold. Then there exists a unique $x \in X$ such that the action of $\alpha \in \mathfrak{A}$ on $M$ is given by $\alpha m:=\alpha(x) \cdot m(\alpha \in \mathfrak{A}, m \in M)$ and such that $M$ is $A_{x}$-irreducible (and hence $A_{x}$-essential).

We close the paper with two remarks concerning the total space $\mathcal{E}$ and section space $\Gamma(\pi)$ of a Banach bundle $\pi: \mathcal{E} \rightarrow X$. They are surely known, but we include them here for the record. 
Remark 1. Let $\pi: \mathcal{E} \rightarrow X$ be a Banach bundle with fibers $E_{x}(x \in X)$,

and let $S \subset X$ be a dense subset. Then $\mathcal{E}_{S}=\dot{\bigcup}\left\{E_{x}: x \in S\right\}$ is dense in $\mathcal{E}$ when $\mathcal{E}$ is given its bundle topology and $\mathcal{E}_{S}$ is given its relative topology inherited from $\mathcal{E}$. (Proof: Let $z \in E_{x} \subset \mathcal{E}$, and let

$$
\mathcal{T}=\mathcal{T}(U, \sigma, \varepsilon)=\{w \in \mathcal{E}: \pi(w) \in U \text { and }\|w-\sigma(\pi(w))\|<\varepsilon\}
$$

be a tube around $z$, where $U$ is a neighborhood of $x, \sigma \in \Gamma(\pi)$, and $\varepsilon>0$; from Section $2, \mathcal{T}$ is a subbasic neighborhood around $z$ in $\mathcal{E}$. Since $S$ is dense in $X$, there exists $s \in S \cap U$. Evidently, $\sigma(s) \in \mathcal{T} \cap E_{s}$.)

Remark 2. The above observation about density of a set of fibers leads to a reasonable conjecture as to a sort of Stone-Weierstrass result regarding density of a set of section, perhaps something like "let $S \subset X$ be dense, and suppose that $M \subset \Gamma(\pi)$ is a $C(X)$-submodule such that $\{\sigma(s): \sigma \in M\}$ is dense in $E_{s}$ for each $s \in S$. Then $M$ is dense in $\Gamma(\pi)$ ". This conjecture is false. Let $X=[0,1]$, and identify $c_{0}(X)$ with the section space of the spiky bundle $\pi_{0}: \mathcal{E} \rightarrow X$, where $E_{x}=\mathbb{K}$ for all $x \in X$. Set

$$
M=\left\{f \in c_{0}(X): f(x)=0 \text { if } x \text { is irrational }\right\} .
$$

Then $M_{s}=\{f(s): f \in M\}=\mathbb{K}$ for each $s$ in the dense set $S=\mathbb{Q} \cap X$, but clearly $M$ is not dense in $c_{0}(X)$.

\section{Acknowledgments}

The authors thank Professor Massoud Amini of Tarbiat Moharres University, Tehran, Iran for his courtesy in answering their questions concerning, especially, the compatibility relations between algebras which underlie subsequent work on module amenability.

The authors also thank the anonymous reviewer for his detailed and useful critique, which has led to several clarifications and other improvements to this paper.

Finally, as the second-named author enters retirement, he wishes to recognize with gratitude the great influence that his dissertation adviser and coauthor Professor Joseph W. Kitchen, Jr. of Duke University had on his career.

\section{References}

[1] Mart Abel, Mati Abel, and P. Tammo, Closed ideals in algebras of sections, Rend. Circ. Mat. Palermo (2) 59(3) (2010), 405-418.

[2] M. Amini, Module amenability for semigroup algebras. Semigroup Forum 69(2) (2004), $243-254$.

[3] M. Amini, Corrigendum: "Module amenability for semigroup algebras", Semigroup Forum 72(3) (2006), 493.

[4] M. Amini, Personal communication, November, 2020 
[5] M. Amini, A. Bodaghi, and B. Shojaee, Module derivations on commutative Banach modules, Commun. Korean Math. Soc. 35(3) (2020), 895-906.

[6] M. Amini and R. Rezavand, Module nuclearity and module injectivity of $C^{*}$-modules, Bull. Math. Soc. Sci. Math. Roumanie (N.S.) 57(4) (2014), 357-366.

[7] E. Behrends, M-structure and the Banach-Stone theorem, Lecture Notes in Mathematics 736, Springer, Berlin, 1979.

[8] A. Bodaghi, Module amenability of the projective module tensor product, Malays. J. Math. Sci. 5(2) (2011), 257-265.

[9] A. Bodaghi, Semigroup algebras and their weak module amenability, J. Appl. Funct. Anal. 7(4) (2012), 332-338.

[10] A. Bodaghi and M. Amini, Module character amenability of Banach algebras, Arch. Math. (Basel) 99 (4) (2012), 353-365.

[11] F. Cunningham and N. Roy, Extreme functionals on an upper semicontinuous space, Proc. Amer. Math. Soc. 42 (1974), 461-465.

[12] J. Dixmier, $C^{*}$-algebras, North-Holland Mathematical Library 15, North-Holland Publishing Co., Amsterdam-New York-Oxford, 1977.

[13] R. Doran and J. Wichmann, Approximate identities and factorization in Banach modules, Lecture Notes in Mathematics 768, Springer-Verlag, Berlin-New York, 1979.

[14] M. J. Dupré and R. M. Gillette, Banach bundles, Banach modules and automorphisms of $C^{*}$-algebras, Research Notes in Mathematics 92, Pitman (Advanced Publishing Program), Boston, MA, 1983.

[15] J. M. G. Fell and R. S. Doran, Representations of *algebras, locally compact groups, and Banach *-algebraic bundles. Vol. 1. Basic representation theory of groups and algebras, Pure and Applied Mathematics 125, Academic Press, Inc., Boston, MA, 1988.

[16] G. Gierz, Representation of spaces of compact operators and applications to the approximation property, Arch. Math. (Basel) 30(6) (1978), 622-628.

[17] G. Gierz, Bundles of topological vector spaces and their duality, Lecture Notes in Mathematics 955, Queen's Papers in Pure and Applied Mathematics 57, SpringerVerlag, Berlin-New York, 1982.

[18] R. Godement, Théorie générale des sommes continues d'espaces de Banach, C. R. Acad. Sci. Paris 228 (1949), 1321-1323.

[19] A. E. Gutman and A. V. Koptev, On the notion of the dual of a Banach bundle, Siberian Adv. Math. 9(1) (1999), 46-98.

[20] A. Ya. Helemskii, The homology of Banach and topological algebras, Mathematics and its Applications (Soviet Series) 41, Kluwer Academic Publishers Group, Dordrecht, 1989.

[21] K. H. Hofmann, The Dauns-Hofmann theorem revisited, J. Algebra Appl. 10(1) (2011), 29-37.

[22] K. H. Hofmann and K. Keimel, Sheaf-theoretical concepts in analysis: bundles and sheaves of Banach spaces, Banach $C(X)$-modules, in: Applications of sheaves (Proc. Res. Sympos. Appl. Sheaf Theory to Logic, Algebra and Anal., Univ. Durham, Durham, 1977), pp. 415-441, Lecture Notes in Mathematics 753, Springer, Berlin, 1979.

[23] T. Hõim and D. A. Robbins, Some extremal properties of section spaces of Banach bundles and their duals II, Quaest. Math. 26(1) (2003), 57-65.

[24] T. Hõim and D. A. Robbins, Isomorphisms into section spaces of Banach bundles, Quaest. Math. 30(1) (2007), 97-113.

[25] T. Hõim and D. A. Robbins, Spectral synthesis and other results in some topological algebras of vector-valued functions, Quaest. Math. 34(3) (2011), 361-376. 
[26] T. Hõim and D. A. Robbins, Amenability as a hereditary property in some algebras of vector-valued functions, in: Function spaces in analysis, 135-144, Contemp. Math. 645, Americam Mathematical Society, Providence, RI, 2015.

[27] B. E. Johnson, Cohomology in Banach algebras, Memoirs of the American Mathematical Society 127, American Mathematical Society, Providence, RI, 1972.

[28] I. Kaplansky, The structure of certain operator algebras, Trans. Amer. Math. Soc. 70 (1951), 219-255.

[29] J. W. Kitchen, Jr. and D. A. Robbins, Tensor products of Banach bundles, Pacific J. Math. 94(1) (1981), 151-169.

[30] J. W. Kitchen, Jr. and D. A. Robbins, Gelfand representation of Banach modules, Dissertationes Math. (Rozprawy Mat.) 203 (1982), 47 pp.

[31] J. W. Kitchen, Jr. and D. A. Robbins, Sectional representation of Banach modules, Pacific J. Math. 109(1) (1983), 135-156.

[32] J. W. Kitchen, Jr. and D. A. Robbins, Linear algebra in the category of $C(X)$-locally convex modules, Rocky Mountain J. Math. 19(2) (1989), 433-480.

[33] J. W. Kitchen and D. A. Robbins, $C(X)$-modules are one-dimensional: a bundletheoretic proof, Rev. Colombiana Mat. 27(3-4) (1993), 209-211.

[34] W. Paravicini, A note on Banach $C_{0}(X)$-modules, Munster J. Math. 1 (2008), 267278.

[35] H. Pourmahmood-Aghababa, (Super) module amenability, module topological centre and semigroup algebras, Semigroup Forum 81(2) (2010), 344-356.

[36] H. Pourmahmood-Aghababa and A. Bodaghi, Module approximate amenability of Banach algebras, Bull. Iranian Math. Soc. 39(6) (2013), 1137-1158.

[37] M. A. Rieffel, Induced Banach representations of Banach algebras and locally compact groups, J. Functional Analysis 1 1967), 443-491.

[38] D. A. Robbins, Some extremal properties of section spaces of Banach bundles and their duals, Int. J. Math. Math. Sci. 29(10) (2002), 563-572.

Wilkes Honors College, Florida Atlantic University, Jupiter, FL 33458 USA

E-mail address: terjehill@fau.edu

5012 N. Via Velazquez, Tucson, AZ 85750 USA; Trinity College, Hartford, CT 06106 USA

E-mail address: david.robbins@trincoll.edu 